CERN-TH/2001-364

\title{
Strangeness, Equilibration, Hadronization
}

\author{
Johann Rafelski \\ Department of Physics, University of Arizona, Tucson, AZ 85721 \\ and \\ CERN-Theory Division, 1211 Geneva 23, Switzerland
}

\begin{abstract}
In these remarks I explain the motivation which leads us to consider chemical nonequilibrium processes in flavor equilibration and in statistical hadroniziation of quark-gluon plasma (QGP). Statistical hadronization allowing for chemical non-equilibrium is introduced. The reesults of fits to RHIC-130 results, including multistrange hadrons, are shown to agree only with the model of an exploding QGP fireball.
\end{abstract}

Submitted to: J. Phys. G: Nucl. Phys. Proceedings of Strange Quark Matter 2001, Frankfurt

\section{Historical background}

The topic we discuss today, production of hadrons in statistical hadronization in high energy heavy ion collisions, has been the subject of several diploma and doctor thesis at the University Frankfurt in late 70's and early 80's. At the time, in a field void of any experimental result, I competed with a Hungarian hadrochemistry group lead by the chair of this discussion session, Prof. J. Zimanyi.

We both passed through natural evolution stages. We were at first considering the equilibrium particle abundances expected to arise when Mr. EquilibriX holds in his hand hot and dense hadron matter fireball, which evaporated these particles. This work was followed by the development of kinetic theory of strangeness production, which was precipitated by the finding of the Budapest group, that light quark interactions were not fast enough to produce strangeness abundantly.

We learned to appreciate that the yields of newly produced quarks were not necessarily given by the chemical equilibrium statistical model. The physics reason which lead us to explore the kinetic theory was relatively short available reaction time in relativistic heavy ion collisions, in general not allowing for the achievement of an equilibrium particle abundance. These remarks apply to the case that the dense matter fireball is made of hadrons, or deconfined quarks. Our work was extended to compare these two so different matter phases.

This kinetic theory work has inspired the chemical non-equilibrium study of hadronization of quark-gluon plasma (QGP), and we were able to show that in a sudden hadronization process specific properties of QGP will be imparted in hadronization on particle yields. All these developments were then summarized in a relatively widely known theoretical review [1].

When the first multistrange hadron experimental results arrived 10 years ago, the analysis applied introduced the chemical non-equilibrium [2], which then was generally accepted as being an important element of data analysis. This analysis addressed solely 
the strange particle abundances. When results for non-strange hadrons were included and were analyzed, it became necessary to expand the chemical-non-equilibrium approach, allowing the abundances of light quarks to vary [3, 歫. In the analysis of the $158 \mathrm{~A} \mathrm{GeV} \mathrm{Pb}$ collisions with $\mathrm{Pb}$ targets, we discovered that the light quark overabundance converged towards the maximum value allowed, at which point the entropy content of the final hadronic state is maximized [5].

In short, we expected chemical nonequilibrium, excess of entropy (hadron multiplicity), and abundant strange antibaryons. Experiments, too numerous to cite, but present in this volume, found these interesting signatures of new physics, just as predicted. So why are we having this discussion? As we evolved in our detailed understanding of the subject, we could see in our research field the arrival of new people who were often fast, but sometimes slow, in repeating our evolutionary steps.

This is not the place to give those who followed the credit for not reading our work. However, we need to deal with the current situation that our early rather trivial work of 1979-81 is today taken seriously again, and is used as basis of data analysis and interpretation. Even if this happens without citation, this is not changing the problem we are facing: if this work is now widely reported, is this 'majority' vote suggesting that the very extensive theoretical development carried out by many prominent theorists over the past 20 years has gone in the wrong direction? Clearly, this is not the case, so we have at least to make an effort to end this rather absurd situation.

The principal speaker (K. Redlich) has presented his views, which we address in technical manner in our main contribution to this volume [6]. This contribution is a qualitative discussion of approaches and results in the study of the experimental data.

\section{Strangeness Equilibration}

Collision of small elementary systems produce colored partons linked by strings, which 'snap' giving birth to particle pairs, and strangeness is in $\mathrm{p}-\mathrm{p}$ interactions produced $1 / 5$ as often compared to the light flavor $\mathrm{u}$ or $\mathrm{d}$. The reasons this happens are understood in the realm of string tension and particle tunneling. Such results form the basis of simulations of nuclear collisions with independent string mechanics.

The formation of a deconfined region of space-time is at origin of another effective mechanism of strangeness production. Beside (confined or deconfined) quarks, we now also have gluons in the hot gas, and glue-glue collisions are believed to be the source of the abundant strangeness observed in the experiment. The string mechanism uses the chromo-electric field between color charges, the gluon is the transverse quantum of the chromo-fields. Thus, though in both cases mechanisms specific to the glue filed are invoked, both mechanisms are complementary.

It is a mere coincidence that given the strength of the string tension $(1 \mathrm{GeV} / \mathrm{fm})$, the mass of the strange quark $(160 \pm 50 \mathrm{MeV})$, and the initial temperatures we reach in the hot fireball $(200-300 \mathrm{MeV})$, that in the $\mathrm{p}-\mathrm{p}$ interactions and central $\mathrm{Pb}-\mathrm{Pb}$ interactions the yield of strangeness is near to chemical equilibrium yield. A lot of theoretical effort went to understand this situation within kinetic models. This fact cannot be generalized to different collision systems without prior and thorough study of the kinetic production mechanism applicable. The extension of the work on strangeness is the study of light quark abundance. It is not guaranteed that at all conditions, and for all systems considered even $u, d$ quarks are found in chemical equilibrium. 


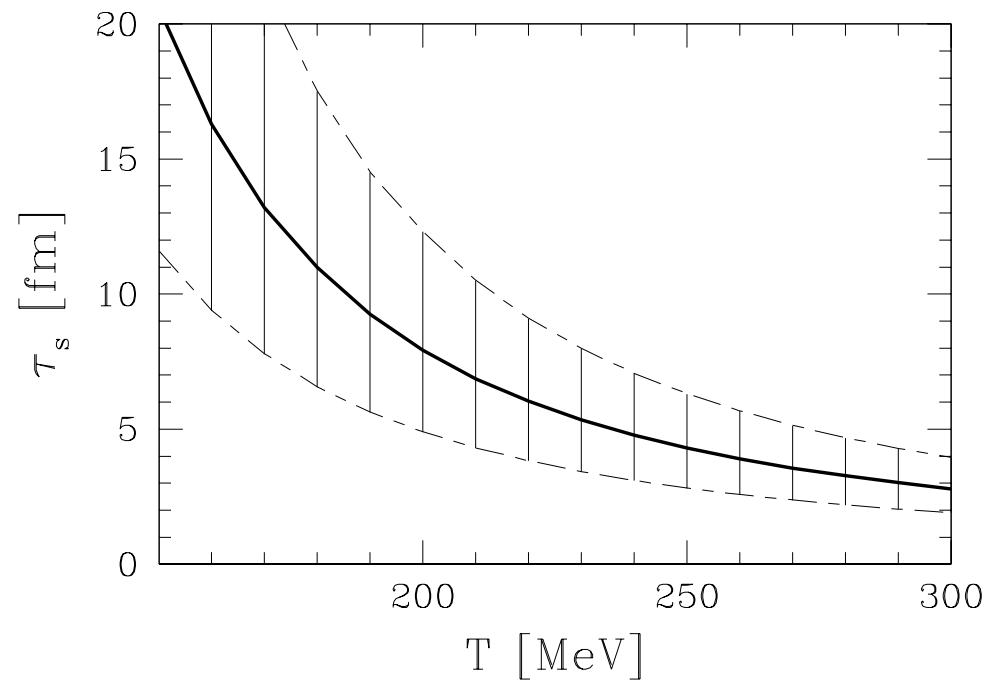

Figure 1. Strangeness chemical relaxation time $\tau_{\mathrm{s}}$, for $\alpha_{s}\left(M_{\mathrm{Z}}\right)=0.118$ with $m_{\mathrm{s}}\left(M_{\mathrm{Z}}\right)=$ $90 \mathrm{MeV}, \rho_{\mathrm{s}}^{\infty}\left(m_{\mathrm{s}} \simeq 200 \mathrm{MeV}\right)$ (thick line). Hatched areas: effect of variation of strange quark mass by $20 \%$.

The deviations from chemical equilibrium can be incorporated into the momentum distribution of (strange) quarks and antiquarks in the deconfined matter in the form:

$$
f_{\mathrm{s} / \overline{\mathrm{s}}}=\frac{1}{\gamma_{\mathrm{s}}^{-1} \lambda_{\mathrm{s}}^{\mp 1} e^{\sqrt{p^{2}+m_{\mathrm{s}}^{2}} / T}+1} \quad \rightarrow \gamma_{\mathrm{s}} \lambda_{\mathrm{s}}^{ \pm 1} e^{-\sqrt{p^{2}+m_{\mathrm{s}}^{2}} / T}
$$

When $\gamma_{\mathrm{s}} \rightarrow 1$ we are approaching chemical equilibrium distribution. On the right hand side in Eq. (1), we see the Boltzmann limit. This distribution maximizes the entropy at given particle number. When a Boltzmann momentum distribution is established, the phase space occupancy $\gamma_{\mathbf{s}}(t)$ may be still quite different from unity, as indicated it evolves in time $t$ due to ongoing pair production (gluon fusion) processes, GG $\rightarrow \mathrm{s} \overline{\mathrm{s}}$.

The characteristic time required to equilibrate strangeness chemically in the deconfined gluon gas is shown Fig.1. This result suggests that depending on the initial temperature and subsequent evolution, the lifespan of the QGP needs to live at high temperature in excess of $4 \mathrm{fm} / \mathrm{c}$ in order for the strange quark flavor to reach full chemical equilibrium. This lifespan of the plasma phase is not generally assured, and thus in QGP the chemical equilibrium is not easily reached.

\section{Hadronization}

Hadrons are always the most abundant final particles observed experimentally. We must be able to understand and interpret the production mechanism of these particles. Strange hadrons are the largest particle family, and are thus particularly interesting. Our primary objective is to identify what changes can be expected in the yields of strange particles emitted from deconfined quark-gluon matter. And we must device a mechanism of data analysis that is sufficiently sensitive to recognize this.

For now nearly 50 years we know that the abundances of hadrons produced in elementary and heavy ion collisions can be, within a factor of two, described by the statistical phase space. Hagedorn [8], and before him, Fermi [9], Landau [10], have 
pioneered that idea. One can argue that when the production probability (the square of the quantum matrix element) of different hadronic particles is similar, they saturate by strength of their interactions the unitarity limit, the primary difference in particle yield is arising from the relative magnitude of the accessible emission phase space.

This reduces the issue to the question, why can we use a 'temperature' $T$ parameter to characterize the size of hadronic particle phase space? Hagedorn spoke of a pre-existent temperature, later he developed a bootstrap model which helped quantify this idea [11]. Another possible approach uses the quark-vacuum fluctuations to obtain Boltzmann-like hadron momentum distribution [12]. In nuclear collisions, a large number of quarks and gluons within a deconfined local domain of space time can approach by two body collision processes the local thermal distribution within a relatively short period of time. In both cases, the confining vacuum is the critical input factor, as it keeps together these strongly interacting quanta. In my view, the confining vacuum makes strong interactions different and introduces the statistical phase space into hadronic particle abundances.

Two properties, both associated with gluons in the deconfined state, allow to remember the structure of the fireball, and eventually help us understand the properties of QGP. The addition of gluonic degrees of freedom is doubling the entropy content of quarks alone, which already benefit from the breaking of color bonds to increase in the deconfined state the entropy content; gluon based reactions are effective in producing strange quarks. The enhancement of the entropy content is seen in enhanced pion yields, while enhanced strangeness abundance contributes to a pattern of strange hadron which favors production of multistrange hadrons.

What is the level of sensitivity in particle detection which is required for these observables? The entropy enhancement translates into a 30-60\% increased pion yield. Strangeness enhancement is expected at the level of factor two-three. One would imagine, seen the magnitude of these effects of QGP, that we need to measure these quantities at the level of a few percent. Thus, we must obtain experimental results at this level of precision, and device an analysis method which is sensitive at this precision level.

In quantitative terms, the relative number of final state hadronic particles freezing out from, e.g., a thermal quark-gluon source, is obtained noting that the fugacity $f_{i}$ of the $i$-th emitted composite hadronic particle containing $k$-components is derived from fugacities $\lambda_{k}$ and phase space occupancies $\gamma_{k}$ :

$$
N_{i} \propto e^{-E_{i} / T_{\mathrm{f}}} f_{i}=e^{-E_{i} / T_{\mathrm{f}}} \prod_{k \in i} \gamma_{k} \lambda_{k} .
$$

As seen in Eq. (2), we study particle production in terms of five statistical parameters $T, \lambda_{\mathrm{q}}, \lambda_{\mathrm{s}}, \gamma_{\mathrm{q}}, \gamma_{\mathrm{s}}$. The difference between the two types of chemical parameters, $\lambda_{i}$ and $\gamma_{i}$, is that the phase space occupancy factor $\gamma_{i}$ regulates the number of pairs of flavor ' $i$ ', and hence applies in the same manner to particles and antiparticles, while fugacity $\lambda_{i}$ applies only to particles, while $\lambda_{i}^{-1}$ is the antiparticle fugacity, as is seen in Eq. (1).

To describe the shape of spectra, one needs matter flow velocity parameters, these become irrelevant when total particle abundances are studied, obtained integrating all of (the velocity deformed) phase space. In presence of strong longitudinal flow, the 'scaling' in rapidity implies that when we are looking at a yield per unit of rapidity, we can also ignore the velocity parameter when considering particle ratios.

The resulting yields of final state hadronic particles are most conveniently characterized taking the Laplace transform of the accessible phase space. This 
approach generates a function which, in its mathematical properties, is identical to the hadron gas partition function:

$$
\mathcal{L}\left[e^{-E_{i} / T_{\mathrm{f}}} \prod_{k \in i} \gamma_{k} \lambda_{k}\right] \propto \ln \mathcal{Z}^{\mathrm{HG}} .
$$

This characterization of the accessible phase space does not require formation of a phase comprising a gas of hadrons, but is not inconsistent with such a step in evolution of the matter. The final particle abundances, measured in an experiment, are obtained after all unstable hadronic resonances ' $j$ ' are allowed to disintegrate, contributing to the yields of stable hadrons. The unnormalized particle multiplicities are obtained differentiating Eq. (3) with respect to particle fugacity.

Are we sure that all particle yields produced predominantly in the statistical hadronization process? Already in 1982, we were pointing out that rarely produced particles such as $\Omega, \bar{\Omega}$ would be the first to show additional enhancement originating in either new mechanisms, or simply in the fact that strangeness correlation would be favoring strange clusters which alter statistical hadronization yields of $\Omega, \bar{\Omega}$ [13.

It is important that the reader understands the method how one finds such exceptions from the statistical hadronization pattern. Given that we have many available particle yields, we can look at the data fits omitting one result at a time, in order to see that the fit is stable, and that it does not depend on one single experimental result. In order to report statistical significance, this procedure must be carried out, it is not implemented in fit programs.

The omission of the $\Omega$ yields (and to a lesser extend $\bar{\Omega}$ ) in the study of SPS results at $\sqrt{s_{\mathrm{NN}}}=17.2 \mathrm{GeV}$, had the effect of allowing for a much better convergence of the statistical hadronization fit. This means that within the reported $\Omega$ (and $\bar{\Omega}$ ) particle yields there are additional contributions outside of the statistical hadronization picture. Subsequent study of the $m_{\perp}$-spectra of $\Omega+\bar{\Omega}$ have in fact confirmed that there is an additional soft momentum component 14]. There are several explanations today of this behavior. Whatever is the true mix of production mechanisms, we simply do not include the $\Omega, \bar{\Omega}$ yields in the statistical hadronization analysis, keeping in mind that their large yield is a challenge for cascade model simulations.

\section{Hadron data fits}

We turn rightaway to discuss the RHIC data obtained in $\sqrt{s_{\mathrm{NN}}}=130 \mathrm{GeV}$ run. The experimental results we consider are the (combined where possible) data of STAR, PHENIX, BRAHMS, PHOBOS, for more discussion of the data origin, see [17. For comparison purposes, we adopted the results nearly as presented in this reference. However, we did not fit natural results such as $\pi^{+} / \pi^{-}=1$. We also do not use the results for $\mathrm{K}^{*}, \overline{\mathrm{K}}^{*}$ since these yields depend on the degree of rescattering of resonance decay products. We assume, in our fits in table 1, that the multistrange weak interaction cascading $\Xi \rightarrow \Lambda$, in the STAR result we consider [15], is cut by vertex discrimination and we use these yields without a further correction.

The data analysed are at the central rapidity region where, due to approximate longitudinal scaling, the effects of flow cancel and we can evaluate the full phase space yields in order to obtain particle ratios. In the last column in table 1, the chemical equilibrium fit, the large $\chi^{2}$ originates in the inability to account for multistrange $\bar{\Xi}, \Xi$. Similar results as here shown [16, are presented in Ref. [17], which work was done before the multistrange hadron yields were reported. 
Table 1. Fits of central rapidity hadron ratios for RHIC $\sqrt{s_{\mathrm{NN}}}=130 \mathrm{GeV}$ run. Top section: experimental results, followed by chemical parameters, physical property of the phase space (energy $E$ and entropy $S$ per baryon number b), and the fit error. Columns: data, full non-equilibrium fit, nonequilibrium fit constrained by strangeness conservation and supersaturation of pion phase space, and in the last column, equilibrium fit constrained by strangeness conservation, upper index ${ }^{*}$ indicates quantities fixed by these considerations.

\begin{tabular}{|c|c|c|c|c|}
\hline & $\overline{\text { Data }}$ & Fit & $\begin{array}{c}\text { Fit } \\
\mathrm{s}-\overline{\mathrm{s}}=0\end{array}$ & $\begin{array}{c}\text { Fit }^{\text {eq }} \\
\mathrm{s}-\overline{\mathrm{s}}=0\end{array}$ \\
\hline$\overline{\mathrm{p}} / \mathrm{p}$ & $0.64 \pm 0.07$ & 0.637 & 0.640 & 0.587 \\
\hline$\overline{\mathrm{p}} / \mathrm{h}^{-}$ & & 0.068 & 0.068 & 0.075 \\
\hline $\bar{\Lambda} / \Lambda$ & $0.77 \pm 0.07$ & 0.719 & 0.718 & 0.679 \\
\hline$\Lambda / \mathrm{h}^{-}$ & $0.059 \pm 0.001$ & 0.059 & 0.059 & 0.059 \\
\hline $\bar{\Lambda} / \mathrm{h}^{-}$ & $0.042 \pm 0.001$ & 0.042 & 0.042 & 0.040 \\
\hline $\bar{\Xi} / \Xi$ & $0.83 \pm 0.08$ & 0.817 & 0.813 & 0.790 \\
\hline$\Xi^{-} / \Lambda$ & $0.195 \pm 0.015$ & 0.176 & 0.176 & 0.130 \\
\hline$\overline{\Xi^{-}} / \bar{\Lambda}$ & $0.210 \pm 0.015$ & 0.200 & 0.200 & 0.152 \\
\hline $\mathrm{K}^{-} / \mathrm{K}^{+}$ & $0.88 \pm 0.05$ & 0.896 & 0.900 & 0.891 \\
\hline $\mathrm{K}^{-} / \pi^{-}$ & $0.149 \pm 0.020$ & 0.152 & 0.152 & 0.145 \\
\hline $\mathrm{K}_{\mathrm{S}} / \mathrm{h}^{-}$ & $0.130 \pm 0.001$ & 0.130 & 0.130 & 0.124 \\
\hline$\Omega / \Xi^{-}$ & & 0.222 & 0.223 & 0.208 \\
\hline $\bar{\Omega} / \overline{\Xi^{-}}$ & & 0.257 & 0.256 & 0.247 \\
\hline $\bar{\Omega} / \Omega$ & & 0.943 & 0.934 & 0.935 \\
\hline$T$ & & $158 \pm 1$ & $158 \pm 1$ & $183 \pm 1$ \\
\hline$\gamma_{q}$ & & $1.55 \pm 0.01$ & $1.58 \pm 0.08$ & $1^{*}$ \\
\hline$\lambda_{\mathrm{q}}$ & & $1.082 \pm 0.010$ & $1.081 \pm 0.006$ & $1.097 \pm 0.006$ \\
\hline$\gamma_{\mathrm{s}}$ & & $2.09 \pm 0.03$ & $2.1 \pm 0.1$ & $1^{*}$ \\
\hline$\lambda_{\mathrm{s}}$ & & $1.0097 \pm 0.015$ & $1.0114^{*}$ & $1.011^{*}$ \\
\hline$E / b[\mathrm{GeV}]$ & & 24.6 & 24.7 & 21 \\
\hline$s / b$ & & 6.1 & 6.2 & 4.2 \\
\hline$S / b$ & & 151 & 152 & 131 \\
\hline$E / S[\mathrm{MeV}]$ & & 163 & 163 & 159 \\
\hline$\chi^{2} /$ dof & & $2.95 /(10-5)$ & $2.96 /(10-4)$ & $73 /(10-2)$ \\
\hline
\end{tabular}

The chemical equilibrium fit yields $E / S=159 \mathrm{MeV}<T=183 \mathrm{MeV}$, and thus in order to satisfy the constraints arising from the Gibbs-Duham relation:

$$
\frac{E}{S}+\frac{P V}{S}=T+\delta T, \quad \delta T=\mu_{\mathrm{b}} \frac{\mathrm{b}}{S}, \quad \mu_{\mathrm{b}}=3 T \ln \lambda_{\mathrm{q}}
$$

the emission volume must be unrealistically large, since the pressure is much smaller than the energy density $E / V$. This inconsistency is to best of my knowledge well known to the promoters of the equilibrium hadronization model, yet they do nothing to resolve it.

On the other hand, in the chemical nonequilibrium fits with $\gamma_{\mathrm{s}}, \gamma_{\mathrm{q}}>1$ there is good agreement with experimental data. Results are as would be expected for a rapidly expanding fireball, they converge to the maximum allowed value:

$$
\gamma_{\mathrm{q}}=\gamma_{\mathrm{q}}^{\mathrm{c}}=e^{m_{\pi} / 2 T_{\mathrm{f}}},
$$


which corresponds to the maximum entropy in the pion gas. The large values of $\gamma_{\mathrm{q}}>1$ is consistent with the need to hadronize the excess entropy of the QGP. The volume problem we addressed above is absent since $E / S=163>T=158 \mathrm{MeV}$. The value of the hadronization temperature $T=158 \mathrm{MeV}$ is below the central expected equilibrium phase transition temperature, and this hadronization temperatures at RHIC is consistent with sudden breakup of a supercooled QGP fireball. We conclude that as has been predicted 15 years ago the inclusion of the yields of multistrange antibaryons in the RHIC data analysis, along with allowance for chemical nonequilibrium $(\gamma \neq 1)$, has the sensitivity to discriminate the different reaction scenarios.

The value of the thermal energy content $E / b=25 \mathrm{GeV}$, seen in table 1, is in very good agreement with expectations once we allow for the kinetic energy content associated with longitudinal and transverse motion. The energy of each particle is 'boosted' with the factor $\gamma_{\perp}^{v} \cosh y_{\|}$. For $v_{\perp}=c / \sqrt{3}$, we have $\gamma_{\perp}^{v}=1.22$. The longitudinal flow range is about \pm 2.3 rapidity units, according to PHOBOS results. To obtain the energy increase due to longitudinal flow, we have to multiply by the average, $\int d y_{\|} \cosh y_{\|} / y_{\|} \rightarrow \sinh (2.3) / 2.3=2.15$, for a total average increase in energy by factor 2.62 , which takes the full energy content to $E^{v} / b \simeq 65 \mathrm{GeV}$ as expected. This consistency reassures that we have a physically relevant fit of the data, which respects overall energy conservation.

The strangeness content, $s / b=6$, is slightly below the expected equilibrated QGP phase space, which would have yielded 8.6 strange quark pairs per baryon at $\lambda_{\mathrm{q}}=1.08$. Thus $\gamma_{\mathrm{s}}^{\mathrm{QGP}}=6 / 8.6 \simeq 0.7$, which is greater than the value 0.5 a similar discussion yields for the SPS energy range. Using the fitted value $\gamma_{\mathrm{s}}^{\mathrm{HG}}=2.1$, we find at RHIC like at SPS, $\gamma_{\mathrm{s}}^{\mathrm{HG}} / \gamma_{\mathrm{s}}^{\mathrm{QGP}} \simeq 3$, which is the correct phase space size ratio for HG and QGP at these conditions.

\section{Final remarks}

There is ample evidence, looking at particle spectra at RHIC, that the evolution of the system is very rapid. We have pioneered this reaction model in the analysis of the SPS data. We have in particular pointed out that the explosive disintegration of the QGP accompanied by supercooling below equilibrium phase transition temperature is a natural phenomenon [18]. Thus, in the hadronization model, we study this is the natural background against which we make decisions how to proceed and how to interpret the results obtained in analysis of particle spectra.

In sudden hadronization, $V^{\mathrm{HG}} \simeq V^{\mathrm{QGP}}$, the growth of volume is negligible, $T^{\mathrm{QGP}} \simeq T^{\mathrm{HG}}$, the temperature is maintained across the hadronization front, and the chemical occupancy factors in both states of matter accommodate the different magnitude of the particle phase space. In this case, the QGP strangeness when 'squeezed' into the smaller HG phase space results in $\gamma_{\mathrm{s}}^{\mathrm{HG}} / \gamma_{\mathrm{s}}^{\mathrm{QGP}} \simeq 3$, which is of the same magnitude as the unfrozen color degeneracy. This theoretical expectation is observed both at the top SPS and at the RHIC-130 run.

For the top SPS energy range, this interesting result could be ignored, since accidentally $\gamma_{\mathrm{s}}^{\mathrm{HG}} \simeq \gamma_{\mathrm{q}}^{\mathrm{HG}}$. For this reason hadronization at SPS energy can be also modeled in terms of an equilibrium hadronization model: The pion enhancement associated with the high entropy phase can be accommodated by use of two temperatures, one for the determination of absolute particle yields, and another for determination of the spectral shape. Such an approach has similar number of parameters, as the method presented here, the only inconsistency (with HBT) is the 
large volume required.

However, already at RHIC-130 the hadron phase space occupancy for strangeness is significantly larger than for light quarks, see table 1. It is the inclusion of the yields of multistrange antibaryons in the RHIC data analysis, which leads to clear resolution of the hadronization mechanism. The specific per baryon strangeness yield at RHIC is an order of magnitude greater than at SPS.

We see at RHIC considerable convergence of the hadron production around properties of suddenly hadronizing entropy and strangeness rich QGP. There is overwhelming theoretical and experimental evidence that the chemical nonequilibrium yields of hadrons are required to understand with the necessary precision the nature of the quark-gluon fireball formed in these interactions.

\section{Acknowledgments}

I would like to thank Horst Stöcker for the organization of this stimulating discussion session at SQM2001 in Frankfurt, and the invitation to present these remarks. Work supported in part by a grant from the U.S. Department of Energy, DE-FG0395ER40937. Laboratoire de Physique Théorique et Hautes Energies, University Paris 6 and 7, is supported by CNRS as Unité Mixte de Recherche, UMR7589.

\section{References}

[1] P. Koch, B. Müller, and J. Rafelski, 1986. Strange quarks in relativistic nuclear collisions. Phys. Rep., 142, 167.

[2] J. Rafelski, 1991. Strange antibaryons from quark-gluon plasma. Phys. Lett. B, 262, 333.

[3] J. Letessier and J. Rafelski, 1999. Chemical non-equilibrium and deconfinement in $200 A$ GeVSulphur induced reactions. Phys. Rev. C, 59, 947.

[4] J. Letessier and J. Rafelski, 1999. Chemical non-equilibrium in high energy nuclear collisions. $J$. Phys. G Nucl. Part. Phys., 25, 295.

[5] J. Letessier and J. Rafelski, 2000. Observing quark-gluon plasma with strange hadrons. Int. J. Mod. Phys. E, 9, 107.

[6] J. Rafelski and J. Letessier, 2002. Importance of reaction volume in hadronic collisions: Canonical enhancement. J. Phys. G Nucl. Part. Phys., this volume; hep-ph/0112151.

[7] J. Rafelski and B. Müller, 1982. Strangeness production in the quark-gluon plasma. Phys. Rev. Lett., 48, 1066. See: Phys. Rev. Lett., 56, 2334E (1986).

[8] R. Hagedorn, 1965. Statistical thermodynamics of strong interactions at high energies. Suppl. Nuovo Cimento, 3, 147.

[9] E. Fermi, 1950. High-energy nuclear events. Prog. Theo. Phys., 5, 570.

[10] L.D. Landau, 1953. On the multiparticle production in high-energy collisions. Izv. Akad. Nauk SSSR, Ser. Fiz., 17, 51. Reprinted in English translation in Collected Papers of L.D. Landau, D. Ter Haar, editor. Pergamon, Oxford, 1965.

[11] R. Hagedorn. Statistical bootstrap model. In Cargese Lectures in Physics, volume 5. Pergamon Press, Oxford, 1973.

[12] A. Bialas, 1999. Fluctuations of the string tension and transverse mass distribution. Phys. Lett. $\mathrm{B}, 466,301$.

[13] J. Rafelski, 1982. Formation and observables of the quark gluon plasma. Phys. Rep., 88, 331.

[14] G. Torrieri and J. Rafelski, 2001. Search for QGP and thermal freeze-out of strange hadrons. New J. of Phys., 3, 12.

[15] J. Castillo, STAR results, presentation at this meeting.

[16] J. Rafelski and J. Letessier, 2002. Strangeness and Statistical QCD. Nucl. Phys. Proceedings Supplement in press. Proceedings of Statistical QCD meeting held at Bielefeld, August 2001. Report-no: CERN-TH/2001-347 and hep-ph/0112027.

[17] P. Braun-Munzinger, D. Magestro, K. Redlich, and J. Stachel, 2001. Hadron production in $\mathrm{Au}-\mathrm{Au}$ collisions at RHIC. Phys. Lett. B, 518, 41.

[18] J. Rafelski and J. Letessier, 2000. Sudden hadronization in relativistic nuclear collisions. Phys. Rev. Lett., 85, 4695. 